\title{
Dielectric Relaxation for Spherical Molecules in a Crystalline Field: Theory for Two Simple Models
}

\author{
John D. Hoffman and Benjamin M. Axilrod
}

\begin{abstract}
A theory of dielectric relaxation is given for two simple models where the dipoles may occupy orientational sites arranged in three dimensions. In the first model, the dipole may point toward the corners of a regular tetrahedron, and in the second, toward the faces of a cube. Both models are simplified to the extent that only one of the sites can differ in energy from all the others.

The calculations show that these models lead to a discrete set of dielectric relaxation times when the sites are not equivalent. The effect of the set of relaxation times on the characteristics of the dielectric relaxation spectrum is examined. The relaxation times are always sufficiently close together so that the dielectric loss is confined to a single region. However, the separation of the relaxation times is sufficient to cause the dielectric loss peak to be considerably broader than that predicted by the Debye equations. In addition, it is found that the loss maximum will frequently be asymmetrical. The variation of the dielectric constant with temperature, and the breadth and asymmetry of the loss peaks are discussed.
\end{abstract}

\section{Introduction}

Our basic viewpoint is that an anisotropy in the crvstalline field hindering the rotation of a dipole in a lattice can be the source of a set of discrete dielectric relaxation times. This viewpoint has already been explored in some detail for a single-axis rotator. ${ }^{12}$ The main objective of this paper is to show how one type of calculation used with the single-axis rotator can be extended to include two simple models where each dipole in the lattice, by turning about a lattice point, can occupy orientational sites arranged in three dimensions. A model permitting such orientations in space may be a reasonable representation for certain molecular crystals composed of polar molecules that are approximately spherical.

The theory presented here and in the papers mentioned above may be regarded as an extension of

1.J. D. Hoffman and H. G. Pfeiffer, J. Chem. Phys, 22, 132 (1954).

2 J. D. Hoffman, ibid. (in press).

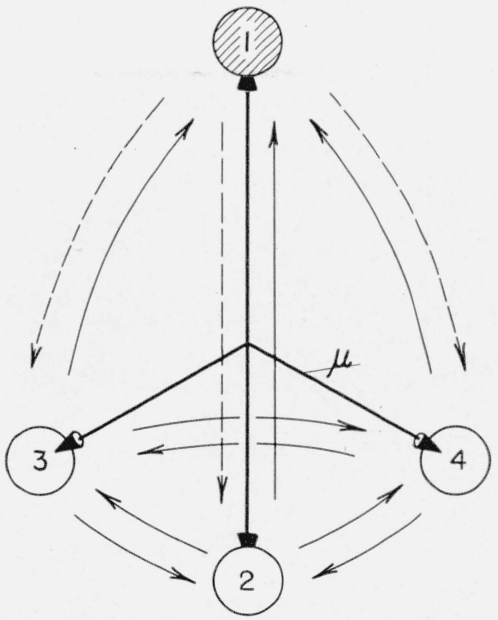

Debye's two-position model. ${ }^{3}$ The two-position model gives only a single ${ }^{134}$ relaxation time; however, a set of relaxation times frequently appears for a single-axis rotator when more than two sites are involved. ${ }^{12}$ We have also modified Debye's theory to the extent that transition probabilities of the elementary process for dipole reorientation have been defined along the lines proposed by Kauzmann. ${ }^{5}$

\section{Tetrahedral Model With One Stable Site}

\subsection{Model}

In this model the dipole possesses four equilibrium orientations (sites) designated 1 through 4 which are arranged in a tetrahedral manner (fig. 1). The permanent dipole moment has the magnitude $\mu$.

3 P. Debye, Polar Molecules, p. 104 (Dover Publications, New York, 1945).
4 H. Fröhlich, Theory of Dielectrics, p. 83 (Oxford University Press, 1949).

5 W. Kauzmann, Rev. Mod. Phys, 14, 12 (1942).

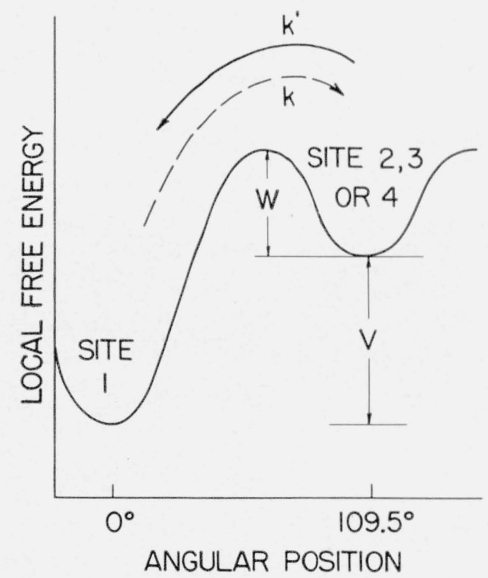

Figure 1. Elementary processes for the tetrahedral model.

Left diagram: Heavy straight arrows indicate equilibrium dipole orientations, light curved arrows the ele mentary processes $k(-\rightarrow \rightarrow)$ and $k^{\prime}(\longrightarrow)$. Site 1 is the stable orientation. Right diagram: Details of the barrier svstem for site 1 relative to sites 2,3 , and 4 . The barrier between sites 2,3 , and 4 (not shown) is $\mathrm{W}$, and the corresponding transition probability is $k^{\prime}$

$340407-55-4$ 
Sites 2, 3, and 4 are equivalent; site 1 is unique, and mav be more stable than the others.

The dipole can turn in a single jump (elementary process) from site 1 to either site 2 , 3 , or 4 by surmounting a local free energy of activation barrier $W+V . \quad W$ and $V$ are shown in figure 1 (right diagram). The barrier system is considered to be the result of the crystalline field imposed on the dipole by its neighbors in the lattice. It is assumed in this simplified treatment that there is no correlation with the motion of neighboring dipoles.

The probability that a dipole will jump from site 1 to either site 2,3 , or 4 is given by

$$
k=B e^{-(W+V) / k T}
$$

where $B$ is a frequency factor, $\boldsymbol{k}$ is Boltzmann's constant, and $T$ the absolute temperature. The dipole must surmount an activation barrier $W$ in order to turn from either site 2 , 3 , or 4 back to site 1 , and the corresponding probability is

$$
k^{\prime}=B e^{-W / k T} .
$$

The barrier between any pair of the sites 2,3 , and 4 is also $W$ so that the probability of the reorientations $2 \rightleftarrows 3,3 \rightleftarrows 4$, and $2 \rightleftarrows 4$ is $k^{\prime}$. It is readily seen that when $V>0$, so that site 1 is relatively stable, we have $k^{\prime}>k$. The assigned transition probabilities for reorientations between the various sites are shown in figure 1 (left diagram.)

\subsection{Rate Equations}

The molecular relaxation times associated with the model are calculated by constructing and solving the differential equations that describe the rate at which dipoles enter and leave each site in terms of the elementary process transition probabilities given by eq (1) and (2). The number of dipoles leaving a site $i$ and entering another designated $j$ is the product of the probability of the transition $i \rightarrow j$ and the population, $N_{i}$, of site $i$. In our problem each $k$ represents the probability that a dipole will leave a site in a specified direction and turn to an adjacent site (single-jump hypothesis, $S-J$ ). ${ }^{1,2}$

Consider the net rate at which dipoles enter and leave site 1 . According to our hypothesis the number per second leaving site 1 and entering site 2 is $k N_{1}$; the total number leaving to enter sites 2,3 , and 4 is $3 k N_{1}$. Similarly, the number returning from site 2 to site 1 is $k^{\prime} N_{2}$. The corresponding numbers leaving sites 3 and 4 to enter site 1 are $k^{\prime} N_{3}$ and $k^{\prime} N_{4}$. Hence the net rate for site 1 is given by $d N_{1} / d t=-$ (number leaving) + (number entering $)=-3 k N_{1}+k^{\prime}\left(N_{2}+N_{3}+N_{4}\right)$. Similar reasoning leads to the differential equations for the net rates for the other sites. Thus we find

$$
\left.\begin{array}{l}
d N_{1} / d t=-3 k N_{1}+k^{\prime}\left(N_{2}+N_{3}+N_{4}\right) \\
d N_{2} / d t=k N_{1}-3 k^{\prime} N_{2}+k^{\prime}\left(N_{3}+N_{4}\right) \\
d N_{3} / d t=k N_{1}+k^{\prime} N_{2}-3 k^{\prime} N_{3}+k^{\prime} N_{4} \\
d N_{4} / d t=k N_{1}+k^{\prime}\left(N_{2}+N_{3}\right)-3 k^{\prime} N_{4} \cdot
\end{array}\right\}
$$

The solutions of eq (3) will provide the molecular (intrinsic) relaxation times, $\tau_{\beta}$, where $\beta$ is the mode of decay, and will also give considerable information regarding the nature of the modes of decay.

\subsection{Relaxation Times and Modes of Decay}

Solutions of the equations of the type illustrated in eq (3) are of the form ${ }^{1.2 .6}$

$$
N_{i}=\sum_{\beta} C_{i \beta} \psi_{\beta} ; \quad i=1,2, \cdots \Omega,
$$

where $i$ refers to a particular site, $\Omega$ is the number of sites, $\beta$ is the mode of decay, and $\psi_{\beta}$ is a decay function of the form

$$
\psi_{\beta}=e_{\beta}^{f}{ }^{\left(k, k^{\prime}\right) t} .
$$

The set of discrete relaxation times is given by

$$
\tau_{\beta}=-1 / f_{\beta}\left(k, k^{\prime}\right) \text {. }
$$

The solutions of eq (3) are obtained by forming the characteristic determinant of the differential equations, and solving for eigenvalues of the operator $\mathbf{D}=d / d t$. These eigenvalues, which are always found to be negative or zero, are the $f_{\beta}\left(k, k^{\prime}\right)$. One of the $f_{\beta}\left(k, k^{\prime}\right)$ is always zero, and this mode is designated by $\beta=1$; the corresponding $C_{i 1}$ are the equilibrium values of the $N_{i}$. The relationships between the $C_{i \beta}$ for each mode where $\beta \geqq 2$ are determined by substituting each solution $C_{i \beta} \psi_{\beta}$ into eq (3). On carrying out the above steps we find that the solutions of eq (3) may be written ${ }^{7}$

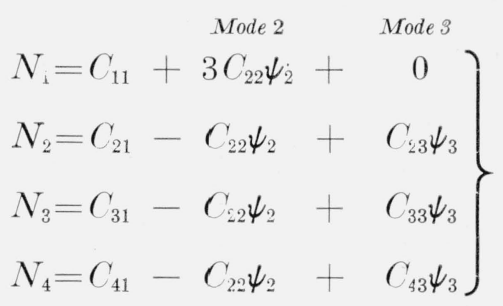

where $\psi_{2}=\exp \left[-\left(k^{\prime}+3 k\right) t\right]$ and $\psi_{3}=\exp \left(-4 k^{\prime} t\right)$. Also, we may note that $C_{21}=C_{31}=C_{41}=C_{11} \exp (-V /$ $\boldsymbol{k} T$ ), and that $C_{23}+C_{33}+C_{43}=0$.

Equation (7) describes the rate at which equilibrium is established after the abrupt removal of a disturbance at $t=0$. In the present case the displacement from equilibrium at $t \leqq 0$ will be assumed to be the result of a static electric field. The quantities $C_{i 2}$ and $C_{i 3}$ in eq (7) represent the (small) number of dipoles that are displaced from their equilibrium orientations by the field.

If we form the vectors $\vec{C}_{i 2}$ where each $\overrightarrow{\boldsymbol{C}}_{i 2}$ has the direction of a dipole in the $i$ th site and the magnitude

\footnotetext{
6 Ince, Ordinary Differential Equations, p. 144 (Dover Publications, New York, 1944).

7 The eigenvalue of the characteristic determinant $\mathbf{D}=-4 k^{\prime}$ occurs twice. In the case of such doubly degenerate roots we actually have $N_{i}=C_{i 1}+C i_{2} \psi_{2}+C_{i 3} \psi_{3}$ $+t C_{i 4} \psi_{3}$. However, it is found by substituting this solution into the original differential equations that the $C_{i 4}$ are identically zero.
} 
$C_{i 2}$, the sum of the $\overrightarrow{\boldsymbol{C}}_{i 2}$ is a measure of the polarization associated with mode 2 . A similar relation applies to mode 3. Thus, eq (7) indicates that, owing to the return of the displaced dipoles to their equilibrium orientations, the polarization will decay with time according to

$$
P(t)=P_{2} e^{-\left(k^{\prime}+3 k\right) t}+P_{3} e^{-4 k^{\prime} t} .
$$

$P_{2}$ and $P_{3}$ are the polarizations associated with modes 2 and 3 at $t=0$. The actual values of $P_{2}$ and $P_{3}$ are calculated in a following section. Since the dielectric relaxation time is defined as the time the polarization takes to fall to $1 / e$ times its original value, it is seen that the molecular relaxation times are

$$
\left.\begin{array}{l}
\tau_{2}=1 /\left(k^{\prime}+3 k\right) \\
\tau_{3}=1 / 4 k^{\prime}
\end{array}\right\}
$$

in accord with eq (6).

Information regarding the nature of the modes of decay can also be obtained from the solutions. The constants $C_{i 2}$ in eq (7) show that mode 2 promotes the attainment of equilibrium by permitting a flow of dipoles from site 1 equally to sites 2,3 , and 4 (or vice versa). Similarly, it is seen that mode 3 involves reorientations among the equivalent sites only; site 1 is not involved. The uncertainty (which is removed when we place an electric field on the system) regarding the exact nature of the motions for mode 3 is a result of the fact that this mode is degenerate.

\subsection{Polarization and Orientational Polarizability Associated With the Relaxation Times}

It is desirable to know the polarization and polarizability associated with each mode of decay.

This means that we must evaluate the $C_{i \beta}$ in eq (7) for $\beta \geqq 2$. To do this we first calculate the new equilibrium number of dipoles, $C_{i 1}^{F}$, in each site when a static electric field $\overrightarrow{\boldsymbol{F}}$ is present, where $F$ is the magnitude of the field.

According to the Boltzmann distribution law we may write

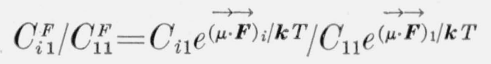

where $C_{i 1}^{F}$ is the equilibrium number of dipoles in site $i$ in the presence of the field $\overrightarrow{\boldsymbol{F}}$, and $C_{11}^{F}$ is the corresponding number for site 1 . The quantity $\overrightarrow{\mu \cdot \boldsymbol{F}}$ is given by

$$
\begin{aligned}
(\overrightarrow{\mu \cdot \boldsymbol{F}})_{i}= & \mu F\left[\sin \xi_{i}^{\prime} \cos \theta_{i}^{\prime} \sin \xi \cos \theta+\right. \\
& \left.\sin \xi_{i}^{\prime} \sin \theta_{i}^{\prime} \sin \xi \sin \theta+\cos \xi_{i}^{\prime} \cos \theta\right] .
\end{aligned}
$$

The angles $\xi_{i}^{\prime}$ and $\theta_{i}^{\prime}$ define the angular position of the $i$ th site, while $\xi$ and $\theta$ define the field direction (fig. 2). After calculating the ratio $C_{i 1}^{F} / C_{11}^{F}$ for $i=2$, 3 , and 4 , and applying the condition $\Sigma_{i} C_{i 1}^{F}=N$ where $N$ is the total number of dipoles in the lattice, we obtain

$$
\left.\begin{array}{rl}
C_{11}^{F}= & C_{11}+4 C_{11} C_{21} \mu F \cos \xi / N \boldsymbol{k} T \\
C_{21}^{F}= & C_{21}-4 C_{11} C_{21} \mu F \cos \xi / 3 N \boldsymbol{k} T- \\
& C_{21} \mu F \sin \lambda \sin \xi(\cos \theta+\sqrt{3} \sin \theta) / 2 \boldsymbol{k} T \\
C_{31}^{F}= & C_{21}-4 C_{11} C_{21} \mu F \cos \xi / 3 N \boldsymbol{k} T- \\
& C_{21} \mu F \sin \lambda \sin \xi(\cos \theta-\sqrt{3} \sin \theta) / 2 \boldsymbol{k} T \\
C_{41}^{F}= & C_{21}-4 C_{11} C_{21} \mu F \cos \xi / 3 N \boldsymbol{k} T+ \\
& C_{21} \mu F \sin \lambda \sin \xi \cos \theta / \boldsymbol{k} T
\end{array}\right\}
$$

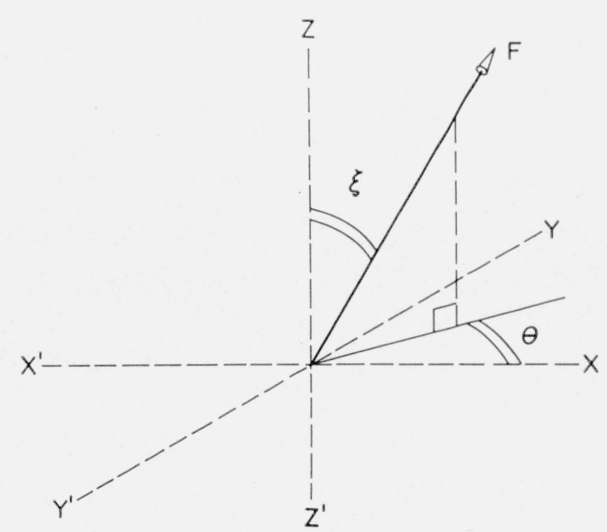

FIGURE 2. Arrangements of the sites and field direction for the tetrahedral model.

Left diagram: Heavy arrows represent the equilibrium dipole orientations. Right diagram: Polar coordinates used to define the field direction. The same angles with the notation $\xi_{i}^{\prime}$ and $\theta_{i}^{\prime}$ are used to define the equilibrium dipole orientations. 
to an excellent approximation. ${ }^{8} \quad \lambda$ is the tetrahedral angle.

The actual number of dipoles displaced by the field into each site for each mode is readily obtained if we note, as implied in sections 2,3 , that

$$
\left.\begin{array}{l}
N_{i}=C_{i 1}^{F} \text { at } t=0 \\
N_{i}=C_{i 1} \text { at } t=\infty .
\end{array}\right\}
$$

With the $C_{i 1}^{F}$ given in eq (12), application of these boundary conditions to eq (7) yields

$$
\left.\begin{array}{l}
C_{12}=4 C_{11} C_{21} \mu F \cos \xi / 3 N \boldsymbol{k} T \\
C_{23}=-C_{21} \mu F \sin \lambda \sin \xi(\cos \theta+\sqrt{3} \sin \theta) / 2 \boldsymbol{k} T \\
C_{33}=C_{21} \mu F \sin \lambda \sin \xi(\sqrt{3} \sin \theta-\cos \theta) / 2 \boldsymbol{k} T \\
C_{43}=C_{21} \mu F \sin \lambda \sin \xi \cos \theta / \boldsymbol{k} T
\end{array}\right\}
$$

Now that the coefficients $C_{i \beta}$ have been evaluated for the case of an applied electric field, the polarization and polarizability associated with each mode at $t=0$ may be calculated. For each mode we obtain the components of the polarization in the $x, y$, and $z$ directions with

$$
\left.\begin{array}{l}
P_{x}=\mu \sum N_{i} \sin \xi_{i}^{\prime} \cos \theta_{i}^{\prime} \\
P_{y}=\mu \sum N_{i} \sin \xi_{i}^{\prime} \sin \theta_{i}^{\prime} \\
P_{z}=\mu \sum N_{i} \cos \ddots_{i}^{\prime}
\end{array}\right\}
$$

where the $N_{i}$ (at $t=0$ ) are given by eq (7) after relations (1.4) have been inserted. To obtain $P_{x}$, $P_{y}$, and $P_{z}$ for a given mode, only the part of the $N_{i}$ involving that mode is employed. The components of $P_{x}, F_{y}^{\prime}$, and $P_{z}$ in the field direction are

$$
\left.\begin{array}{l}
P_{(x, F)}=P_{x} \sin \xi \cos \theta \\
P_{\left(y, F^{*}\right)}=P_{y} \sin \xi \sin \theta \\
P_{(z, F)}=P_{z} \cos \xi .
\end{array}\right\}
$$

The average value of the polarization for all orientations of the applied field is given by

$$
P_{\beta}=\bar{P}_{(x, F)}+\bar{P}_{(y, F)}+\bar{P}_{(z, F)} .
$$

The averaging over all orientations of the applied field implies that the sample is polycrystalline.

Following the above procedure we obtain for mode 2

$$
P_{2}=\left(\frac{16 C_{11} C_{21}}{3 N}\right)\left(\frac{\mu^{2} F}{3 \boldsymbol{k} T}\right)
$$

\footnotetext{
${ }^{8}$ In applying eq $(10)$, the exponentials have been expanded and terms $O \vec{O}(\overrightarrow{\boldsymbol{F}})$ $\boldsymbol{k} T)^{2}$ and higher dropped. This is permissible since for ordinary field strengths $\overrightarrow{\mu \cdot \boldsymbol{F}} / \boldsymbol{k} T<<1$.
}

and for mode 3

$$
P_{3}=\left(\frac{8 C_{21}}{3}\right)\left(\frac{\mu^{2} F}{3 \boldsymbol{k} T}\right)
$$

These are the polarizations referred to in eq (8). Using the defining relation $P_{\beta}=N \alpha_{\beta} F$, we find the molecular orientational polarizabilities to be

$$
\begin{aligned}
& \alpha_{2}=\left(\frac{16 C_{11} C_{21}}{3 N^{2}}\right)\left(\frac{\mu^{2}}{3 \boldsymbol{k} T}\right)=\frac{16}{3}\left[\frac{k k^{\prime}}{\left(k^{\prime}+3 k\right)^{2}}\right]\left(\frac{\mu^{2}}{3 \boldsymbol{k} T}\right), \\
& \alpha_{3}=\left(\frac{8 C_{21}}{3 N}\right)\left(\frac{\mu^{2}}{3 \boldsymbol{k} T}\right)=\frac{8}{3}\left[\frac{k}{\left(k^{\prime}+3 k\right)}\right]\left(\frac{\mu^{2}}{3 \boldsymbol{k} T}\right)
\end{aligned}
$$

where we have made use of the fact that $C_{11} / N=k^{\prime} /$ $\left(k^{\prime}+3 k\right)$ and $C_{21} / N=k /\left(k^{\prime}+3 k\right)$. The total polarizability, $\alpha_{\text {total }}, \quad$ is given bv $\left[8 k\left(k^{\prime}+k\right) /\left(k^{\prime}+3 k\right)^{2}\right]$ $\left(\mu^{2} / 3 \boldsymbol{k} T\right)$ or $\left[8 g(1+g) /(1+3 g)^{2}\right]\left(\mu^{2} / 3 \boldsymbol{k} T\right)$ where $g=k / k^{\prime}$.

\subsection{Characteristics of the Dielectric Relaxation Spectrum}

So far, we have given our results in terms of quantities that describe how the polarization will decay with time after the abrupt removal of a static field. It should be noted that the relaxation times and polarizabilities which describe the rate of decay, as well as the relative magnitude of each decay process, are expressed in terms of molecular properties. It is desirable to apply these results to macroscopic systems where the measuring field is sinusoidal so that we may learn something of the shape of the regions of loss in the dielectric relaxation spectrum.

If the polarization of a macroscopic system decays after the abrupt removal of a static field with time according to $\left[\mathrm{cf}\right.$. eq (8) ${ }^{9}$

$$
P(t)=\sum_{\beta} P_{\beta} e^{-t / \mathbf{T}_{\beta}},
$$

where $\mathrm{T}_{\beta}$ is the macroscopic relaxation time for the $\beta$ th mode of decay, we may write (assuming linear superposition) for sinusoidal fields

$$
\epsilon^{\prime \prime}(\omega)=\sum_{\beta} \Delta \epsilon_{\beta} \omega \mathrm{T}_{\beta} /\left(1+\omega^{2} \mathrm{~T}_{\beta}^{2}\right) .
$$

$\epsilon^{\prime \prime}(\omega)$ is the dielectric loss factor, $\omega$ the angular frequency, and $\Delta \epsilon_{\beta}$ the increment of dielectric constant associated with the $\beta$ th mode of decay. Equation (19b) reduces to the form of the Debye equa$\operatorname{tion}^{10}$ for $\epsilon^{\prime \prime}(\omega)$ when there is only one relaxation time. For an Onsager-like internal field, it has been suggested by Powles ${ }^{11}$ that $\mathrm{T}_{\beta} \cong \tau_{\beta}$, and we will employ this approximation. Also, for this field it is reasonable for the purposes of an approximate analysis to assume that $\Delta \epsilon_{\beta} \cong K \cdot \alpha_{\beta}$ where $K$ is a con-

8 The macroscopic relaxation time exceeds the molecular relaxation time owing to the tendency of the internal field to maintain itself, and the magnitude of the effect is proportional to the difference between the applied field $E$ and the internal field $F$.

${ }_{10}$ See p. 94 of reference in footnote 3

11 J. G. Powles, J. Chem. Phys, 21, 633 (1953). 
stant. ${ }^{12}$ With these approximations we have the working equation, ${ }^{12,2}$

$$
\epsilon^{\prime \prime}(\omega) \cong K \cdot \sum_{\beta} \alpha_{\beta} \omega \tau_{\beta} /\left(1+\omega^{2} \tau_{\beta}^{2}\right)
$$

This equation, together with eq (9) and (18) can be used to predict the general characteristics of the dielectric relaxation spectrum for the tetrahedral model. For the present, we will restrict the discussion to the nature of a plot of $\epsilon^{\prime \prime}$ against $\log \omega$. This discussion will be facilitated by reference to table 1 where the ratios $\alpha_{2} / \alpha_{3}$ and $\tau_{2} / \tau_{3}$ are given, together with values for the total orientational polarizability, $\alpha_{\text {total }}$, for various values of $k / k^{\prime}$. It is convenient to discuss the results in terms of what happens as the ratio $k / k^{\prime}$ goes from zero to unity.

When site 1 is relatively stable so that $k / k^{\prime}$ is small compared to unity, it is seen from the table that the relaxation times are moderately far apart, and that the polarizabilities are unequal but still of comparable magnitude. Under these conditions the dielectric loss peak calculated with eq (20) is not only broader than that predicted by the Debye equation with a single relaxation time, but is also slightly asymmetrical. Since the longer relaxation time, $\tau_{2}$, is associated with the larger polarizability, $\alpha_{2}$, the loss peak is somewhat steeper on the low frequency side (fig. 3, curve A). Since the total polarizability is rather low when $k / k^{\prime}$ is small, that part of the dielectric constant resulting from orientation of dipoles will be small.

If we now raise $k / k^{\prime}$ to the value one-third, the relaxation times are somewhat closer together than before, and the polarizabilities are equal. The calculated loss maximum is still somewhat broader than one would predict from the Debye equation, and is also symmetrical (fig. 3, curve B).

TABLE 1. Properties of the tetrahedral model

\begin{tabular}{|c|c|c|c|}
\hline$g=k / k^{\prime}$ & $\begin{array}{l}\text { Ratio of the } \\
\text { relaxation } \\
\text { times } \tau_{2} / \tau_{3}\end{array}$ & $\begin{array}{c}\text { Ratio of the } \\
\text { polarizabilities } \\
\alpha_{2} / \alpha_{3}\end{array}$ & $\begin{array}{c}\text { Total polariz- } \\
\text { ability } \alpha_{\text {total }} \\
\text { in units of } \\
\mu^{2} / 3 \boldsymbol{k} T\end{array}$ \\
\hline $\begin{array}{l}0 \\
1 / 100 \\
1 / 10 \\
1 / 3 \\
1 / 2 \\
3 / 4 \\
1\end{array}$ & $\begin{array}{l}4 \\
3.88 \\
3.07 \\
2 \\
1.60 \\
1.23 \\
1\end{array}$ & $\begin{array}{l}2 \\
1.94 \\
1.54 \\
1 . \\
.80 \\
.62 \\
.50\end{array}$ & $\begin{array}{r}0 \\
.08 \\
.52 \\
.89 \\
.96 \\
.99 \\
1\end{array}$ \\
\hline
\end{tabular}

As $k / k^{\prime}$ is increased above a value of one-third, the relaxation times continue to converge so that the loss maximum becomes even narrower. The polarizabilities are again unequal so that the loss maximum again becomes asymmetrical, but owing to the close spacing of the relaxation times the effect will hardly be noticeable. It should be observed that this slight asymmetry is of a type opposite to that found for values of $k / k^{\prime}<1 / 3$.

${ }_{12}$ These approximations are probably fairly accurate, especially in the case where the dielectric constant is low.

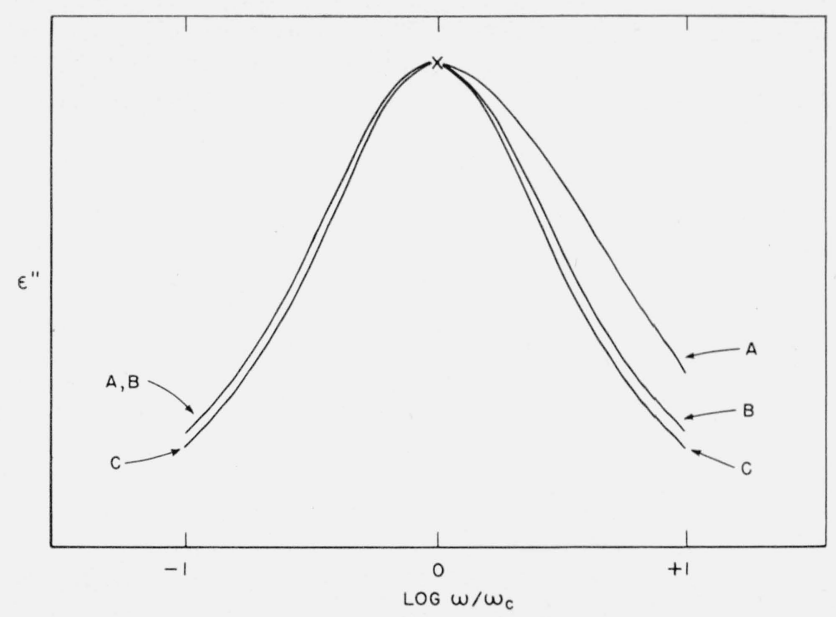

Figure 3. Shape of the dielectric loss peaks for the tetrahedral model.

Curve A, asymmetrical and broadened peak calculated for $g \cong 0$; curve $B$, broadened but symmetrical peak calculated for $g=1 / 3$; curve $\mathrm{C}$, narrow and symmetrical peak for $g=1$ (single relaxation time). $\omega$ is the measuring frequency, metrical peak for $g=1$ (single relaxation time). $\omega$ is the measuring frequency, and $\omega_{c}$ is the frequency where $\epsilon$ is a maximum. For convenience in comparing
the shape of the curves, the maximum value for the loss has been matched at $X$.

Finally, as the ratio $k / k^{\prime}$ tends to unity, the total polarizability reaches the full value $\mu^{2} / 3 \boldsymbol{k} T$, and only a single relaxation time $\tau_{2}=\tau_{3}=1 / 4 k$ remains. Thus, the Debye equation for $\epsilon^{\prime \prime}(\omega)$ holds (fig. 3, curve C). It must be emphasized that, although the polarizability becomes $\mu^{2} / 3 \boldsymbol{k} T$, the value for a free rotator, we are dealing with hindered rotation inasmuch as barriers still exist between the sites. Hence, the observed mean relaxation time for the model will always considerably exceed that of a free rotator.

The dependence of the shape, breadth, and magnitude of the loss maximum with increase of temperature is easily obtained if we note that

$$
g=k / k^{\prime}=e^{-V / \boldsymbol{k} T}
$$

It is clear from this expression that the ratio $k / k^{\prime}$ will increase with rising temperature even if $V$ is a constant. Therefore, the previous discussion of the changes in the dielectric relaxation spectrum for the case of increasing values of $k / k^{\prime}$ also holds for the case of rising temperature. Thus, for the tetrahedral model it is seen that the loss maximum will generally tend to become narrower with rising temperature. The loss peak will be steeper on the low frequency side if $k / k^{\prime}$ is between zero and onethird; i. e., if $V / \boldsymbol{k} T$ lies between $\infty$ and 1.1. Hence, for a very large range of temperature one should expect to find some asvmmetry, and this asymmetry should tend to diminish as the temperature is increased. An analysis shows that the total polarizability, and hence the dielectric constant, will rise up to a temperature where $T \cong V / 1.95 \boldsymbol{k}$. Above this temperature the $1 / T$ term in the polarizability begins to predominate so that the total polarizability will fall with further increase of temperature. 


\section{Six-position Model With One Stable Site}

The details of the model are shown in figure 4 . The dipole in any lattice point may lie in either direction along each of the Cartesian axes, $x, y$, and $z$. Site 1 is unique; all the other sites are equivalent and may be unstable with respect to site 1 . The model might correspond, for instance, to the case where the central dipole in a body-centered cubic lattice prefers to point toward one face of the cube, but may also point toward any of the five remaining faces.

The $S-J$ hypothesis is assumed. A dipole can turn from site 1 to any of the adjacent sites $(2,3,4$, or 5) with a probability $k$ where $k$ is defined as in eq (1). All other reorientations to adjacent sites occur with a probability $k^{\prime}$ [eq (2)]. The assigned probabilities for the various elementary processes are shown in figure 4. It should be observed that the double jumps $1 \rightarrow 6,2 \rightarrow 4$, and $3 \rightarrow 5$ (or the reverse processes) are forbidden as elementary processes by the $S-J$ hypothesis.

The rate equations are

$$
\left.\begin{array}{l}
d N_{1} / d t=-4 k N_{1}+k^{\prime}\left(N_{2}+N_{3}+N_{4}+N_{5}\right) \\
d N_{2} / d t=k N_{1}-4 k^{\prime} N_{2}+k^{\prime}\left(N_{3}+N_{5}+N_{6}\right) \\
d N_{3} / d t=k N_{1}+k^{\prime} N_{2}-4 k^{\prime} N_{3}+k^{\prime}\left(N_{4}+N_{6}\right) \\
d N_{4} / d t=k N_{1}+k^{\prime} N_{3}-4 k^{\prime} N_{4}+k^{\prime}\left(N_{5}+N_{6}\right) \\
d N_{5} / d t=k N_{1}+k^{\prime}\left(N_{2}+N_{4}\right)-4 k^{\prime} N_{5}+k^{\prime} N_{6} \\
a N_{6} / d t=k^{\prime}\left(N_{2}+N_{3}+N_{4}+N_{5}\right)-4 k^{\prime} N_{6} .
\end{array}\right\}
$$

The solutions may be written in the form

$$
\begin{gathered}
N_{i}=C_{i 1}+C_{i 2} \psi_{2}+C_{i 3} \psi_{3}+C_{i 4} \psi_{4}+C_{i 5} \psi_{5} ; \\
\psi_{2}=e^{-4 k^{\prime} t}, \\
\psi_{3}=e^{-6 k^{\prime} t}, \\
\psi_{4}=e^{-\left(2 k+3 k^{\prime}-Q\right) t}, \\
\psi_{5}=e^{-\left(2 k+3 k^{\prime}+Q\right) t}, \\
Q=\sqrt{4 k^{2}-8 k k^{\prime}+5 k^{\prime 2}} .
\end{gathered}
$$

Substitution of the solutions $C_{i \beta} \psi_{\beta}$ for $\beta \geqq 2$ in the differential equations yields the following: ${ }^{13}$

Mode 2.

$$
\left.\begin{array}{l}
C_{12}=C_{62}=0 \\
C_{22}=-C_{42} \\
C_{32}=-C_{52} .
\end{array}\right\}
$$

This mode of decay thus involves only those dipoles reorienting in sites $2,3,4$, and 5 . The mode is active in the dielectric relaxation spectrum. ${ }_{13}$ Mode 2 is doubly degenerate, and is handled in the same manner as modes
3 and 4 for the tetrahedral model.

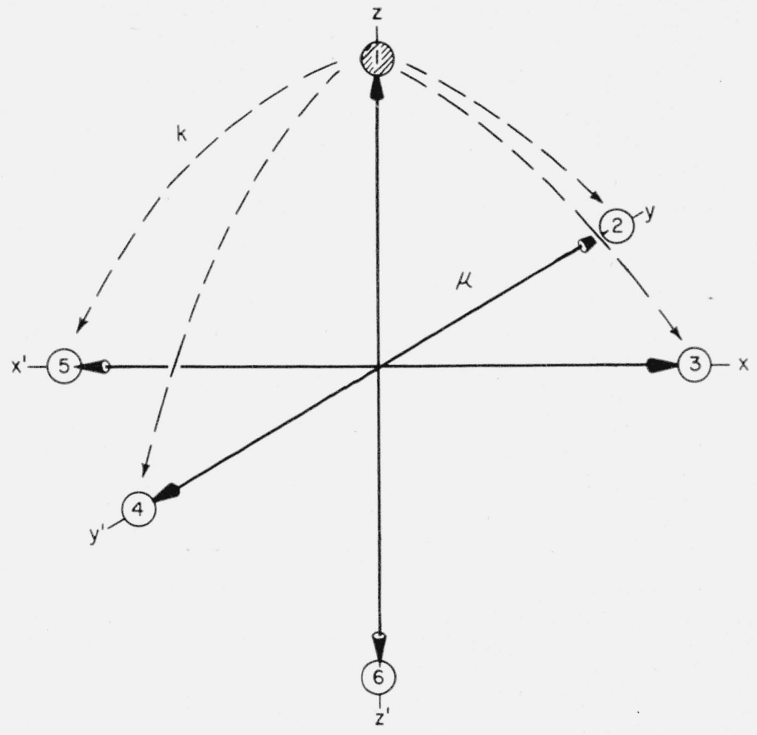

FIgure 4. Six-position model with a single stable orientation (site 1).

Heavy straight arrows indicate the equilibrium dipole orientations. Light dashed arrows indicate elementary processes which take place with a probability v. All other jumps to adjacent sites (such as $2 \rightarrow 1$ or $2 \rightarrow 3$ ) take place with a probability $\%^{\prime}$. Jumps of the type $1 \rightarrow 6$ or $5 \rightarrow 3$ are forbidden as elementary processes. The coordinates used to denote the field direction and orientations processes. The coordinates used to denote the field direction an
of the sites are the same as those given in figure 2 (right diagram).

Mode 3.

$$
\left.\begin{array}{l}
C_{13}=C_{63}=0 \\
C_{23}=-C_{33}=C_{43}=-C_{53} .
\end{array}\right\}
$$

Again, only dipoles in the plane containing sites 2, 3,4 , and 5 are involved. This mode of decay is inactive in the dielectric relaxation spectrum owing to a compensatory motion of the dipoles; the sum of the vectors $\overrightarrow{\boldsymbol{C}}_{i 3}$ is zero, so no polarization results. A similar situation arises for the single-axis rotator with four equivalent sites. ${ }^{1}$

Mode 4.

$$
\left.\begin{array}{l}
C_{24}=C_{34}=C_{44}=C_{54} \\
C_{14}=-4(1-1 / H) C_{24} \\
C_{64}=-4 C_{24} / H
\end{array}\right\}
$$

where $H=2 g-1-q, q=Q / k^{\prime}$, and $g=k / k^{\prime}$.

Mode 5.

$$
\left.\begin{array}{l}
C_{25}=C_{35}=C_{45}=C_{55} \\
C_{15}=-4\left(1-1 / H^{\prime}\right) C_{25} \\
C_{65}=-4 C_{25} / H^{\prime}
\end{array}\right\}
$$

where $H^{\prime}=2 g-1+q$. Both modes 4 and 5 are complicated and involve dipoles in all of the sites.

The polarizability associated with each mode of decay is readily calculated in the same manner as for the tetrahedral model. We summarize the 
results below together with the relaxation times.

$$
\begin{gathered}
\left.\alpha_{2}=\left(\frac{4 g}{1+5 g}\right)\left(\frac{\mu^{2}}{3 \boldsymbol{k} T}\right), \quad \begin{array}{c}
\tau_{2}=1 / 4 k^{\prime} ; \\
\tau_{3}=1 / 6 k^{\prime} ;
\end{array}\right\} \\
\alpha_{3}=0, \quad \frac{g(q+3-2 g)[(2 g-1+q)(2 g+1)-2(g-1)]}{(1+5 g)^{2} q}\left(\frac{\mu^{2}}{3 \boldsymbol{k} T}\right), \\
\alpha_{4}=1 /\left(2 k+3 k^{\prime}-Q\right) ; \\
\alpha_{5}=\frac{g(2 g-3+q)[(2 g-1-q)(2 g+1)-2(g-1)]}{(1+5 g)^{2} q}\left(\frac{\mu^{2}}{3 \boldsymbol{k} T}\right), \\
\tau_{5}=1 /\left(2 k+3 k^{\prime}+Q\right) .
\end{gathered}
$$

The total polarizability is given by $[12 g(1+2 g) /(1+$ $\left.5 g)^{2}\right]\left(\mu^{2} / 3 \boldsymbol{k} T\right)$. Since the results are rather complicated, we resort to calculation of the polarizabilities and relaxation times numerically for various values of $k / k^{\prime}$. The results are given in table 2 .

It is convenient to discuss the results in terms of what happens as $k / k^{\prime}$ goes from zero to unity. It is seen in table 2 that the maximum separation of the relaxation times occurs when $k / k^{\prime}$ tends toward zero. We then have $\tau_{4} / \tau_{5} \cong 6.85$. The two most important relaxation times, $\tau_{2}$ and $\tau_{4}$, tend to converge as $k / k^{\prime}$ increases, with the result that the corresponding dielectric loss maximum will at first be somewhat broader than a Debye type peak, but becomes more narrow as $k / k^{\prime}$ increases, and finally assumes the simple Debye form when $k=k^{\prime}$. The total polarizability always increases with increasing $k / k^{\prime}$.

As in the case of the tetrahedral model, the loss peaks will be noticeably asymmetrical for low values of $k / k^{\prime}$. Owing to the fact that the longest relaxation time is associated with the largest polarizability when $k / k^{\prime}<0.2$, the loss maximum will be steeper on the low frequency side in this region. At about $k / k^{\prime}=0.2$ the peak will be slightly broadened but nearly symmetrical since the dominant polarizabilities are equal. A slight asymmetry again appears when $k / k^{\prime}$ exceeds $\sim 0.2$, but it will not be particularly noticeable owing to the closeness of the relaxation times. It should be observed that the slight asym-

\begin{tabular}{|c|c|c|c|c|c|c|c|}
\hline \multirow{2}{*}{$g=k / k^{\prime}$} & \multicolumn{3}{|c|}{$\begin{array}{l}\text { Relaxation times } \\
\left.\text { (in units of } 1 / k^{\prime}\right)\end{array}$} & \multicolumn{4}{|c|}{$\begin{array}{l}\text { Polarizabilities } \\
\text { (in units of } \mu^{2} / 3 \boldsymbol{k} T \text { ) }\end{array}$} \\
\hline & $\tau_{2}$ & $\tau_{4}$ & $\tau_{5}$ & $\alpha_{2}$ & $\alpha_{i}$ & $\alpha_{5}$ & $\alpha_{\text {total }}$ \\
\hline $\begin{array}{l}0 \\
1 / 100 \\
1 / 10 \\
1 / 5 \\
1 / 2 \\
1\end{array}$ & $\begin{array}{r}0.25 \\
.25 \\
.25 \\
.25 \\
.25 \\
.25\end{array}$ & $\begin{array}{r}1.31 \\
1.25 \\
.88 \\
.66 \\
.39 \\
.25\end{array}$ & $\begin{array}{r}0.191 \\
.191 \\
.190 \\
.189 \\
.185 \\
.167\end{array}$ & $\begin{array}{l}0 \\
.038 \\
.267 \\
.400 \\
.571 \\
.666\end{array}$ & $\begin{array}{l}0 \\
.069 \\
.347 \\
.404 \\
.377 \\
.333\end{array}$ & $\begin{array}{l}0 \\
.004 \\
.026 \\
.036 \\
.031 \\
0\end{array}$ & $\begin{array}{r}0 \\
.111 \\
.640 \\
.840 \\
.979 \\
1.9\end{array}$ \\
\hline
\end{tabular}
metry that appears in this region is such that the loss peaks will be steeper on the high frequency side. The asymmetry completely disappears when $k=k^{\prime}$.

Table 2.-Properties of the six-position model
The expression $k / k^{\prime}=\exp (-V / \boldsymbol{k} T)$, where $V$ is the energy difference between site 1 and each of the other sites, gives the dependence of $k / k^{\prime}$ on temperature. It follows that $k / k^{\prime}$ will increase with rising temperature, so the discussion given above in terms of growing values of $k / k^{\prime}$ also holds for the case of increasing temperature. The dielectric behavior of this model is qualitatively similar to that of the tetrahedral model. The total polarizability rises up to the temperature $T \cong V / 2.17 \boldsymbol{k}$, but falls as the temperature is increased further.

\section{Discussion}

It is clear from the results cited above that an anisotropy in the crystalline field can lead to the existence of a set of discrete dielectric relaxation times for polar molecules that possess orientational sites arranged in three dimensions. The separation of the relaxation times is a maximum when the crystalline field is highly anisotropic. The relaxation times tend to merge as the temperature is increased.

The following experimental predictions hold for both models: (1) The regions of loss in the dielectric relaxation spectrum will become narrower with increasing temperature. (2) At low temperatures the dielectric loss peak will be steeper on the low frequency side. This asymmetry will decrease as the temperature is raised, and practically completely disappear somewhat before the loss peak assumes the simple Debye form. At high temperatures the Debye equations will be a good approximation. (3) The dielectric constant will be small when the temperature is low, and will increase as the temperature is raised; at temperatures above $T \sim V / 2 \boldsymbol{k}$, the dielectric constant will decrease with rising temperature. It should be noted that no real substance should be expected to show the entire range of behavior mentioned above since melting or transition phenomena may intervene.

It should be observed that the effect of a set of (discrete) active relaxation times on the shape of the loss regions is virtually indistinguishable from the effect of a suitably chosen continuous "distribution" of relaxation times. Also, it should be noted that the effects that have been predicted for the case of rising temperature with $V$ constant will occur in a more marked way if $V$ decreases with rising temperature, as is often assumed for the case of cooperative interaction.

The models treated here are obviously rather specialized, and do not necessarily correspond closely to any real crystal. For instance, one might expect the anisotropy of the crystalline field to be such that more than two transition probabilities are required to represent the system. More general models are being studied.

Washington, January 5, 1955. 